S. Jenisch

S. Koch

T. Henseler

R.P. Nair

J.T. Elder

C.E. Watts

E. Westphal

J.J. Voorhees

E. Christophers

M. Krönke

\section{Corneodesmosin gene polymorphism demonstrates strong linkage disequilibrium with HLA and association with psoriasis vulgaris}

\section{Key words:}

corneodesmosin; HLA antigens; HLA haplotypes; genetics; psoriasis; S-gene

\section{Acknowledgments:}

We gratefully acknowledge the skilled assistance of Herdis Sommer and Martina Wedler for HLA typing, and thank Philip Stuart for critical review of the manuscript. Parts of this research was supported by award DFG-WE 905/1-1 from the German Research Foundation (E.W., T.H., S.J.), by USPHS awards P30 HG00209-03 and R01 AR4274-01 (J.T.E., R.N., J.J.V.), and by the Babcock Memorial Trust (J.T.E., R.N., J.J.V.). J.T.E. is supported by the Department of Veterans Affairs Medical Center, Ann Arbor, Michigan.
Received 29 April, revised,

accepted for publication 22 July 1999

Copyright $\odot$ Munksgaard 1999

Tissue Antigens . ISSN 0001-2815

Tissue Antigens 1999: 54: 439-449

Printed in Denmark . All rights reserved
Abstract: Corneodesmosin (CD) is thought to play a key role in corneocyte cohesion, and its proteolysis appears to be a major event in the process of desquamation. Recently it was shown that CD is encoded by the S-gene, which is located approximately $160 \mathrm{~kb}$ telomeric of HLA-C. In the present study, the role of $\mathrm{CD}$ in the genetics of psoriasis vulgaris was studied in greater detail. The second exon of the CD gene was sequenced in 86 HLAtyped individuals from 13 psoriasis multiplex families. A total of 11 silent dimorphisms and 7 variants resulting in amino acid substitutions were found. Pedigree analysis showed that these variants could be grouped into 7 alleles, encoding 6 different amino acid sequences. These alleles are in strong linkage disequilibrium with HLA-B and -C, indicating that the polymorphism of the $\mathrm{CD}$ gene is ancient and well conserved rather than sporadic. One allele at the $\mathrm{CD}$ locus, designated $\mathrm{CD} 2$, displayed strong linkage disequilibrium with HLA-Cw6, the HLA allele most prominently associated with psoriasis. CD2 demonstrated a greater relative risk than Cw6 (3.4 vs. 2.5, not significant) and higher significant transmission disequilibrium with psoriasis than any of the investigated HLA-alleles. Due to its biologic function, cellular location and disease association, the CD gene appears to be an excellent candidate gene for PSORS1, the HLA-linked determinant of psoriasis vulgaris

Psoriasis vulgaris is a chronic inflammatory and hyperproliferative skin disease affecting approximately $2 \%$ of the Caucasian population (1). Familial psoriasis (type I psoriasis) is characterized by early-onset ( $<40$ years) as compared to late-onset psoriasis (type II psoriasis) which yields a much weaker genetic predisposition (2). Psoriasis demonstrates a strong immunological background (3), displaying strong associations with alleles at several HLA loci (reviewed in (4)). Family studies yielded strong evidence for a psoriasis susceptibility locus within the major histocompatibility complex (MHC), designated PSORS1 (HUGO/GDB-approved symbol) (5-8).

The nucleotide sequences reported in this paper have been submitted to the GenBank ${ }^{\mathrm{TM}} / \mathrm{EBI}$ data bank with accession numbers AJ238461-AJ238467.
Authors' affiliations:

S. Jenisch ${ }^{1}$,

S. Koch ${ }^{1}$,

T. Henseler ${ }^{2}$,

R.P. Nair ${ }^{3}$,

J.T. Elder ${ }^{3,4}$,

C.E. Watts ${ }^{2}$,

E. Westphal ${ }^{1}$,

J.J. Voorhees ${ }^{3}$,

E. Christophers ${ }^{2}$,

M. Krönke ${ }^{1}$

${ }^{1}$ Department of Immunology, University of Kiel, Kiel, Germany,

${ }^{2}$ Department of Dermatology, University of Kiel, Kiel, Germany,

${ }^{3}$ Department of Dermatology, University of Michigan, Ann Arbor, Michigan, USA

${ }^{4}$ Dermatology Service, Department of Veterans Affairs Medical Center, Ann Arbor, Michigan, USA

Correspondence to: Stefan Jenisch, M.D. Institute of Immunology University of Kiel Michaelisstrasse 5 D-24105 Kiel Germany
Tel: +49 4315973380 Fax: +494315973335 e-mail:

jenisch@immunologie.uni-kiel.de 
A

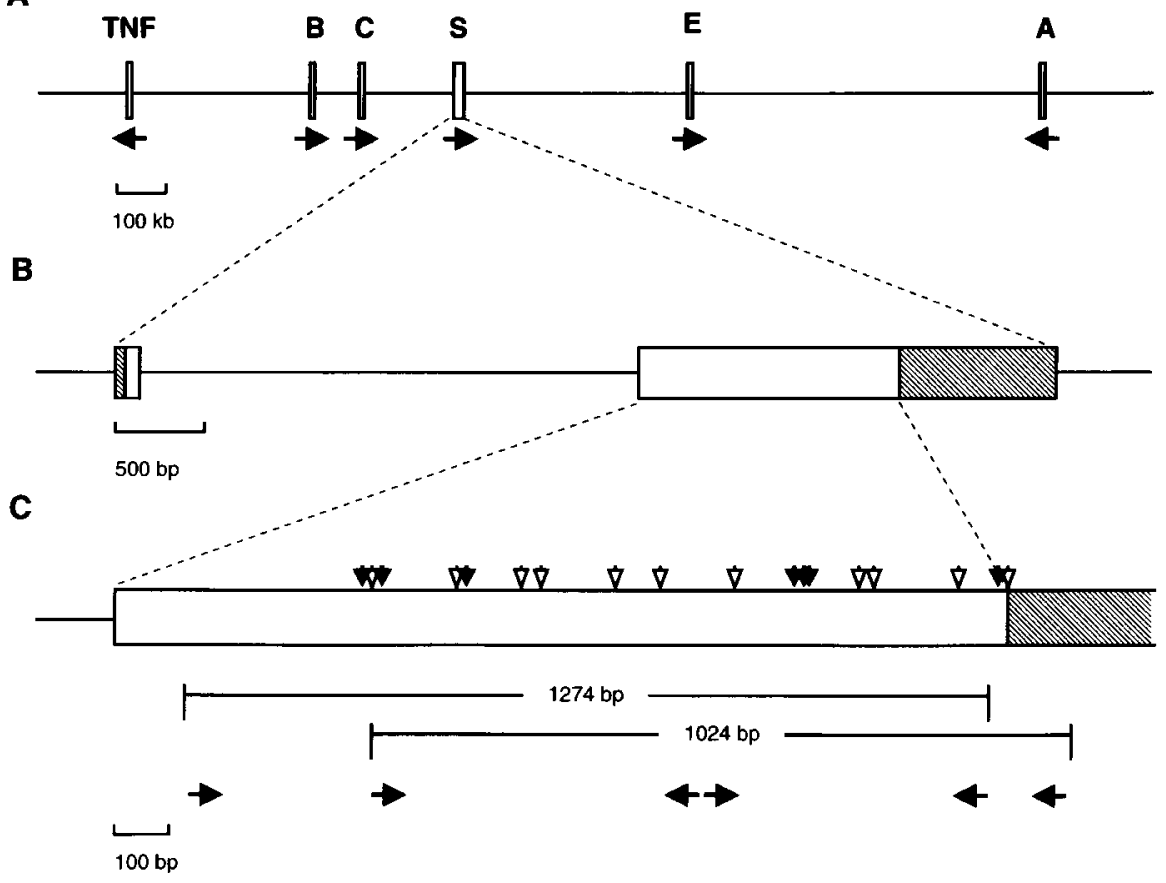

Fig. 1. Genetic organization of the S-gene. (A) The $\mathrm{S}$ gene is located in the HLA-class I region, approximately $160 \mathrm{~kb}$ telomeric from HLA-C. (B) The two exons of the S-gene are separated by a $2.9 \mathrm{~kb}$ intron. The hatched boxes represent the $5^{\prime}$ - and the $3^{\prime}$ - untranslated regions. (C) PCR- and sequencing strategy. The horizontal lines indicate the two PCRs, arrows indicate the localization of the sequencing primers. The polymorhic sites of the S-gene are marked by vertical arrows. (open arrows: silent mutations).
Our previous studies have indicated that the disease-causing allele of PSORS1 exists in linkage disequilibrium with distinct HLAhaplotypes, the most prominent of which is the ancestral haplotype (AH) 57.1 (Cw6-B57-DRB1*0701-DQB1*0303). However, several arguments suggest that the disease-associated HLA alleles are unlikely to encode the disease determinant $(7,9)$.

In 1993, Zhou and Chaplin described a new protein which they proposed to be involved in keratinocyte differentiation (10). This protein was encoded by a gene located within the MHC, approximately 160 kb telomeric of HLA-C (Fig. 1A). It was designated as the S-gene, and proposed as a psoriasis susceptibility locus, due to its location within the MHC (11). In 1997, Haftek et al. independently identified a keratinocyte-specific protein, corneodesmosin (CD) (12), which was subsequently shown to be the protein product of the S-gene (13).

Several functional aspects of CD further recommend the CD gene as a strong candidate for the PSORS1 determinant. Secreted by keratinosomes, $\mathrm{CD}$ is translocated to the extracellular part of the desmosome; in this way it differs from all other desmosomal proteins previously described. Its putative adhesion function seems important for corneodesmosome formation or function. If so, CD could play a key role in the control of corneocyte cohesion. Based on recent evidence, proteolysis of $\mathrm{CD}$ appears to be a major event in the process of corneocyte desquamation (12-14). Thus, studies which identify mutations or allelic polymorphisms of the $\mathrm{CD}$ gene should help to further clarify the genetic background of psoriasis. Within the coding region of the $\mathrm{CD}$ gene, eleven diallelic polymorphic sites have been described to date. Three of the nine dimorphic sites detected in the Japanese population resulted in amino acid substitutions (15), whilst two further mutations found in Caucasians amino lead to amino acid exchanges (13). However, neither study determined whether these variants represented sporadic mutations or belonged to conserved alleles of the $\mathrm{CD}$ gene. Moreover, it is still unclear whether the $\mathrm{CD}$ gene alleles demonstrate linkage disequilibrium with defined HLA haplotypes. Only one study investigated allelic associations between three polymorphic sites of $\mathrm{CD}$ and psoriasis; however, no significant associations were identified (15).

Information regarding $\mathrm{CD}$ gene polymorphisms, the HLA haplotypes carrying these polymorphisms, and their associations with psoriasis is far from complete. A comprehensive catalogue of allelic polymorphism is an important prerequisite for linkage and association studies of the $\mathrm{CD}$ gene. To this end, we have identified $\mathrm{CD}$ gene polymorphisms, identified the alleles which carry them, and investigated whether these alleles might play a direct role in the genetics of psoriasis.

\section{Material and methods}

\section{Ascertainment}

Thirteen families with psoriasis vulgaris of Caucasian origin were investigated. Ten of these were ascertained from probands living 
in northern Germany; the remaining three were ascertained from probands living in southeast Michigan. DNA and HLA data were available from a total of 44 diseased and 42 healthy family members (Table 1). Seven of these families are reported here for the first time; the HLA types of the other families were described in previous investigations $(6,7,9,16,17)$. These families were chosen because their HLA haplotypes could be unambiguously identified and because they displayed clear segregation of the psoriasis phenotype with HLA-B/C haplotypes. These haplotypes included the "classical" Cw6-bearing haplotypes Cw6-B13, Cw6-B57 and Cw6-B37; the Cw8-B65 positive haplotype which was described by us to be a potential psoriasis-risk haplotype (17), and three pedigrees in which rare haplotypes or haplotypes not described to be associated with psoriasis, appeared to segregate with disease.

\section{HLA typing and estimation of HLA haplotypes}

HLA class II and HLA-C typing was performed by DNA-based techniques as described (9). HLA-A and -B were typed either by serology (18) or in ambiguous cases by polymerase chain reaction using sequence-specific primers (PCR-SSP) with a commercially available HLA-typing kit (Dynal Allset ${ }^{\text {TM }}$ SSP-HLA-A, HLA-B). HLA haplotypes were estimated by the use of the HAPCHILD program of the GAS package v2.0 (ㄷ A. Young, Oxford University, 1993-1995) and confirmed by visual inspection of pedigrees.

\begin{tabular}{|c|c|c|c|c|}
\hline Family & Origin & Generations & $\begin{array}{l}\text { Individuals } \\
\text { (affected/healthy) }\end{array}$ & Haplotypes \\
\hline 1 & German & 3 & $6(3 / 3)$ & 5 \\
\hline 2 & German & 3 & $4(3 / 1)$ & 5 \\
\hline 3 & German & 3 & $15(7 / 8)$ & 10 \\
\hline 4 & German & 2 & $5(3 / 2)$ & 4 \\
\hline 5 & German & 3 & $8(3 / 5)$ & 6 \\
\hline 6 & German & 2 & $7(4 / 3)$ & 4 \\
\hline 7 & German & 2 & $6(3 / 3)$ & 4 \\
\hline 8 & German & 2 & $5(2 / 3)$ & 4 \\
\hline 9 & German & 3 & $8(3 / 5)$ & 6 \\
\hline 10 & German & 2 & $5(3 / 2)$ & 4 \\
\hline 11 & US & 3 & $8(5 / 3)$ & 6 \\
\hline 12 & US & 2 & $5(3 / 2)$ & 4 \\
\hline 13 & US & 2 & $4(2 / 2)$ & 4 \\
\hline
\end{tabular}

$\begin{array}{lll}\text { Total } & 86(44 / 42) & 66\end{array}$

The number of haplotypes indicates the number of different HLA-haplotypes observed within the family

Table 1

\section{Analysis of the CD gene DNA-sequence}

The second exon of the $\mathrm{CD}$ gene was amplified by the use of two sets of PCR primer pairs (S303/A and S1555/B, S653/A and 1676/B, Table 2), which cover almost the complete second exon of CD (nt. 303 to nt. 1676, Fig. 1B, C). PCR was performed using standard conditions (19) in a genAmp PCR System 9600 (Perkin-Elmer, Norwalk, NJ, USA). Cycling parameters for PCR were $5 \mathrm{~min}$ at $94^{\circ} \mathrm{C}$ (denaturation) followed by 35 cycles of $20 \mathrm{~s}$ at $94^{\circ} \mathrm{C}, 20 \mathrm{~s}$ at $58^{\circ} \mathrm{C}$ and $60 \mathrm{~s}$ at $72^{\circ} \mathrm{C}$ before a final extension step at $72^{\circ} \mathrm{C}$ for $5 \mathrm{~min}$.

For DNA-sequencing, six primers were used which allowed overlapping sequencing of almost the entire coding region of the second exon (Table 2), (Fig. 1C). DNA-sequencing of the amplified PCR products was performed on an automated DNA sequencer (ABI 373A, Perkin-Elmer) using the fluorescent-dye-terminator technology following the manufacturer's instructions $\left(\mathrm{ABI}\right.$ prism $^{\mathrm{TM}}$ BigDye Terminator Cycle Sequencing Kit; Perkin Elmer). Sequences were analysed using the Sequence-Navigator (Perkin Elmer) and the PC-Gene software (Intelligenetics, MountainView, CA, USA).

\section{Estimation of polymorphic sites and CD alleles}

For the identification of polymorphic sites and alleles of the CD gene, the second exon was sequenced from all 86 probands. The 172 alleles descend from sixty six different chromosomes (Table 1). Variants were identified by inspection of the electrophoretograms and validated by repeated sequencing, and inspection of the inheritance using the haplotype information provided by the HLA data of the pedigrees studied.

For the identification of $\mathrm{CD}$ alleles, the phase of inheritance of each polymorphic site was investigated by analyzing the inheritance of the residues in parent-offspring-trios or multiple sibships. Individual $\mathrm{CD}$ gene sequences were assigned to alleles when the phase of each polymorphic residue was unambiguously clarified.

\section{Estimation of linkage disequilibrium between the CD gene and HLA loci}

CD-HLA haplotypes were determined by careful visual inspection of the pedigrees. Each haplotype was counted once, independent of the number of occurrences in the respective family. Linkage disequilibrium between the $\mathrm{CD}$ gene and each of the HLA-loci was estimated by comparing the number of existing haplotypes with the expected value which was determined by multiplying the observed CD-allele frequencies by the observed HLA-allele frequencies. $\chi^{2}$ values were calculated out of the resulting $i \times j$ contingency tables, with $i=$ number of alleles of the respective HLA-locus and $j=$ num- 


\begin{tabular}{|c|c|c|c|c|}
\hline Primer & $\begin{array}{l}\text { Nucleotide } \\
\text { Position }\end{array}$ & & & Sequence \\
\hline S303/A & $303-320$ & forw & $\mathrm{P}$ & ATT GCC CAG GGT GGT TCT \\
\hline S1555/B & 1555-1576 & rev & $\mathrm{P}$ & GGT AGG AAA ACT TCA GGG TCA G \\
\hline S653/A & $653-671$ & forw & $\mathrm{P} / \mathrm{S}$ & CAA CCA GCG TCC CTG TAG T \\
\hline S1676/B & 1676-1692 & rev & $P$ & СCT ATG CCT GGG CAC TG \\
\hline S311/A & $311-328$ & forw & S & GGG TGG TTC TGC AGG ATC \\
\hline S1204/B & $1204-1223$ & rev & $\mathrm{s}$ & GCT AGA ACT GCT GGG GAC TC \\
\hline $\mathrm{S} 1240 / \mathrm{A}$ & $1240-1258$ & forw & $\mathrm{s}$ & GTT (C/T)AC CCT ACC ATC CCT G \\
\hline S1550/B & 1550-1569 & rev & S & AAA CTT CAG GGT CAG CTA GC \\
\hline S1653/B & $1653-1674$ & rev & s & СCT TCT CCC ATA TGG GAT ATA G \\
\hline
\end{tabular}

All the sequences are shown in the $5^{\prime}$ to $3^{\prime}$ direction. The positions of the primers are given according to the base sequence of $C D$ (13). Forw, rev: forward or reverse primer. P,S: PCR (P) or DNA sequencing (S)

ber of alleles of the CD-locus. $P$-values were estimated from the $\chi^{2}$ values with respect to $(\mathrm{i}-1) \times(\mathrm{j}-1)$ degrees of freedom.

To estimate the allelic association between distinct alleles of the $\mathrm{CD}$ and of HLA-alleles, relative delta $\left(\Delta_{\mathrm{r}}\right)$ values were calculated. For this purpose, the absolute association $\Delta(\mathrm{i}, \mathrm{j})$ between the allele $i$ of the HLA locus and $\mathrm{j}$ of the CD gene was estimated by $\Delta(\mathrm{i}, \mathrm{j})=$ $p(i, j)-p(i) p(j)$, where $p(i, j)$ is the haplotype frequency and $p(i), p(j)$ are the respective gene frequencies. Since these absolute delta values are not comparable, the relative delta values, defined as $\Delta_{\mathrm{r}}(\mathrm{i}, \mathrm{j})=\Delta$ (i,j) / $\Delta_{\max }(\mathrm{i}, \mathrm{j})$, with $\Delta_{\max }(\mathrm{i}, \mathrm{j})=\mathrm{p}(\mathrm{i})(1-\mathrm{p}(\mathrm{j}))$; with $\mathrm{p}(\mathrm{j}) \mathrm{p}(\mathrm{i})$ and $\Delta_{\max } \geq 0$, were calculated (20). The statistical significance of each of these haplotypes was then estimated by Fisher's exact test based upon on the corresponding $2 \times 2$ tables resulting from the presence or absence of the involved alleles.

\section{Association analysis}

Association between psoriasis and $\mathrm{CD}$ alleles, single polymorphic residues of $\mathrm{CD}$, and HLA alleles was estimated by the transmission test for linkage disequilibrium (TDT) (21). In this test, the number of transmitted alleles from parents heterozygous for the marker locus to affected children are compared with the expected value of 0.5. $P$-values were estimated using the resultant $2 \times 2$ contingency tables. An estimate of the relative risk is given by the transmission ratio (number of a particular allele transmitted to an affected offspring divided by the number of times it is not transmitted to an affected offspring). A transmission ratio above one indicates a positive association whilst a transmission ratio below one denotes a negative association.

\section{Results}

\section{Polymorphic sites within the second exon of CD}

Of the 66 independent chromosomes investigated in this study, we observed seventeen dimorphic sites (Table 3). Six dimorphisms resulted in the exchange of an amino acid whilst the deletion of an AGC triplet at nucleotide position (nt.) 468 caused the loss of a serine residue. The insertion of three bases (aag) at nt. 1602, located within the stop codon, did not cause a change in the translated sequence since a new stop codon is created at the same position $\left(\mathrm{t}_{1602} \mathrm{aa}_{1604} \rightarrow \mathrm{t}_{1602}\right.$ aag aa $\left.\mathrm{aa}_{1604}\right)$.

Of the polymorphisms identified, six silent and four functional mutations were previously unreported. One of the polymorphic sites described in the Japanese investigation $\left(\mathrm{g} / \mathrm{t}_{1240}, \mathrm{gly} / \mathrm{val}_{409}\right)(15)$ was not detected during this study.

In all 66 analysed chromosomes we found the additional guanosine $(\mathrm{G})$ at nt. 1521 not identified in the original S-gene sequence (10), but reported for CD (13). The insertion moves the stop-codon from nt. 1515 to nt. 1602 and results in a translated region of CD which is 27 amino acids longer than that predicted for the S-gene product.

\section{CD allele sequences}

In total, seven different $\mathrm{CD}$ sequences were identified, encoding six different proteins (Table 4). Two alleles differed only in a single substitution (c/ $t_{902}$ ) and encode for the same amino acid sequence (Table 5). Six of the CD sequences were observed on at least three 
Mutations in the second exon of the S-gene

\begin{tabular}{|c|c|c|c|c|}
\hline \multicolumn{2}{|c|}{ Nucleotides } & \multicolumn{2}{|c|}{ Amino acids } & \multirow[b]{2}{*}{ Reference } \\
\hline Position & Subst. & Position & Subst. & \\
\hline 442 & $g / a$ & $143(127)$ & ser/asp & - \\
\hline 461 & $a / c$ & & & - \\
\hline 468 & agc/- & $153(137)$ & ser/- & - \\
\hline 614 & $\mathrm{a} / \mathrm{g}$ & & & 15 \\
\hline 619 & $\mathrm{c} / \mathrm{t}$ & $202(186)$ & ser/phe & 15 \\
\hline 722 & $\mathrm{t} / \mathrm{c}$ & & & - \\
\hline 767 & $\mathrm{a} / \mathrm{g}$ & & & 15 \\
\hline 902 & $\mathrm{c} / \mathrm{t}$ & & & - \\
\hline 971 & $\mathrm{t} / \mathrm{c}$ & & & 15 \\
\hline 1118 & $\mathrm{a} / \mathrm{g}$ & & & 13,15 \\
\hline 1215 & $a / g$ & 401 (385) & ser/gly- & - \\
\hline 1236 & $t / g$ & 408 (392) & ser/ala & 13 \\
\hline 1240 & $\mathrm{~g} / \mathrm{t}^{*}$ & 409 (393) & gly/val* & 15 \\
\hline 1243 & $\mathrm{c} / \mathrm{t}$ & 410 (394) & ser/leu & 15 \\
\hline 1331 & $\mathrm{~g} / \mathrm{c}$ & & & - \\
\hline 1358 & $t / c$ & & & - \\
\hline 1515 & $-* / g$ & 501 (485) & & 13 \\
\hline 1593 & $\mathrm{c} / \mathrm{t}$ & $527(511)$ & asp/asn & - \\
\hline 1602 & -/aag & & & - \\
\hline
\end{tabular}

The positions of the mutations are given according to the CD sequence (13). Values in brackets indicate positions according to the S-gene sequence originally published (10). (*) not observed in this study. Subst.: base or amino acid substitutions

Table 3 independent chromosomes, whereas one was seen only in three related individuals. For this report, the alleles encoding different proteins were preliminarily designated as CD1-CD6 and the two sequences encoding the same protein as CD1a and CD1b.

In this study, none of the investigated individuals displayed the S-gene sequence originally published by Zhou and Chaplin (10); nor the CD sequence as described by Guerrin et al. (13). Despite the insertion of a guanosine at nt. 1515, at least one of two other possible sequence divergences with the S-gene sequence (either at nt. $902(\mathrm{c} \rightarrow \mathrm{t})$ or at nt. $1118(\mathrm{a} \rightarrow \mathrm{g}))$ were observed in our cohort. The amino acid sequence of the $\mathrm{CD}$ gene differs from the sequence of the S-gene product by virtue of a substitution of serine to alanine at position 408 and the addition of 27 amino acids. In this study, we failed to observe the corneodesmosin sequence as found by Guerrin et al. (13). Although we also detected ala $_{408}$ in CD4, this allele differs in another position $\left(\mathrm{phe}_{202}\right.$ ) from the original CD-sequence (Table 5).

\section{Linkage disequilibrium between the CD-gene and HLA}

Linkage disequilibrium of the $\mathrm{CD}$ was found to be highly significant with the class I loci HLA-B and -C, only moderately significant with HLA-A and non-significant with HLA-class II (Table 6). A number of strong allelic associations were identified (Table 7). Of the frequently found HLA alleles, $\mathrm{Cw}^{*} 06, \mathrm{Cw}^{*} 0701, \mathrm{Cw} * 0702, \mathrm{~B} 57, \mathrm{~B} 7$ and B8 yielded linkage disequilibrium of either $100 \%$ or almost $100 \%$ with the respective $\mathrm{CD}$ gene alleles, whereas $\mathrm{Cw} * 0303, \mathrm{Cw}^{*} 0304$, $\mathrm{B} 60$ and $\mathrm{B} 62$ yielded only weak allelic associations and were found

\begin{tabular}{|c|c|c|c|c|c|c|c|c|c|c|c|c|c|c|c|c|c|c|c|}
\hline & \multicolumn{18}{|c|}{ Position } & \multirow[b]{5}{*}{$\mathrm{N}$} \\
\hline & 0 & 0 & 0 & 0 & 0 & 0 & 0 & 0 & 0 & 1 & 1 & 1 & 1 & 1 & 1 & 1 & 1 & 1 & \\
\hline & 4 & 4 & 4 & 6 & 6 & 7 & 7 & 9 & 9 & 1 & 2 & 2 & 2 & 3 & 3 & 5 & 5 & 6 & \\
\hline & 4 & 6 & 6 & 1 & 1 & 2 & 6 & 0 & 7 & 1 & 1 & 3 & 4 & 3 & 5 & 1 & 9 & 0 & \\
\hline & 2 & 1 & 8 & 4 & 9 & 2 & 7 & 2 & 1 & 8 & 5 & 6 & 3 & 1 & 8 & 5 & 3 & 2 & \\
\hline S-gene & g & a & agc & a & c & $\mathrm{t}$ & g & c & $\mathrm{t}$ & a & a & $\mathrm{t}$ & c & g & $\mathrm{t}$ & - & c & - & 0 \\
\hline Corneodesmosin & g & a & agc & a & c & $\mathrm{t}$ & g & c & $\mathrm{t}$ & g & a & g & c & g & $\mathrm{t}$ & g & c & - & 0 \\
\hline CD1a & g & a & agc & a & c & $\mathrm{t}$ & g & c & $\mathrm{t}$ & g & a & $\mathrm{t}$ & c & g & $\mathrm{t}$ & g & c & - & $17(40)$ \\
\hline CD1b & g & a & agc & a & c & $\mathrm{t}$ & g & $\mathrm{t}$ & $\mathrm{t}$ & g & a & $\mathrm{t}$ & c & g & $\mathrm{t}$ & g & c & - & $1(3)$ \\
\hline $\mathrm{CD} 2$ & g & a & agc & a & $\mathrm{t}$ & $\mathrm{t}$ & g & c & $\mathrm{t}$ & g & a & $\mathrm{t}$ & c & g & $\mathrm{t}$ & g & c & - & $11(42)$ \\
\hline CD3 & g & a & agc & a & $\mathrm{t}$ & $\mathrm{t}$ & g & c & $\mathrm{t}$ & g & g & $\mathrm{t}$ & c & g & $\mathrm{t}$ & g & c & - & $3(11)$ \\
\hline CD4 & g & a & agc & a & $\mathrm{t}$ & $\mathrm{t}$ & $a$ & c & c & g & a & g & c & g & $\mathrm{t}$ & g & c & - & $6(11)$ \\
\hline CD5 & a & a & agc & g & $\mathrm{t}$ & c & $g$ & c & c & a & a & $\mathrm{t}$ & $t$ & c & $\mathrm{t}$ & g & $\mathrm{t}$ & aag & 16 (34) \\
\hline CD6 & g & c & - & g & $\mathrm{t}$ & c & g & C & C & a & a & $\mathrm{t}$ & $\mathrm{t}$ & c & c & g & c & aag & 12 (31) \\
\hline
\end{tabular}

$n$ : number of observed alleles on independent $(n=66)$ chromosomes. In brackets: total number of chromosomes observed $(n=172)$ 
Amino-acid sequence comparisons of the S-gene alleles

\begin{tabular}{lllllllll}
\hline \multicolumn{1}{l}{} & \multicolumn{1}{l}{ Position } \\
\cline { 2 - 8 } & & & & & & & & \\
\hline S-gene product & ser & ser & ser & ser & ser & ser & stop & - \\
corneodesmosin & ser & ser & ser & ser & ala & ser & asp & asp \\
CD1 & ser & ser & ser & ser & ser & ser & asp & asp \\
CD2 & ser & ser & phe & ser & ser & ser & asp & asp \\
CD3 & ser & ser & phe & gly & ser & ser & asp & asp \\
CD4 & ser & ser & phe & ser & ala & ser & asp & asp \\
CD5 & asp & ser & phe & ser & ser & leu & asp & asn \\
CD6 & ser & - & phe & ser & ser & leu & asp & asp \\
\hline
\end{tabular}

Table 5

linked with as many as three or even four different $\mathrm{CD}$ gene alleles. Conversely, CD2, CD3, CD5 and CD6 were found to be linked to specific HLA-C and -B alleles in nearly all cases, whereas CD1a, CD1b, and CD4 demonstrated a much more balanced distribution (Table 7).

Clear evidence for haplotype-specific linkage disequilibrium was found (Table 8). Without exception, all Cw6-B57 or Cw6-B37 haplotypes were linked with $\mathrm{CD} 2$. Two other Cw6-positive haplotypes (B38, B50), each of which presented once in this study, displayed linkage with CD2. In contrast, Cw6-B13 was linked with CD3, which differs from CD2 in one amino acid $\left(\mathrm{gly}_{401}\right)$. All seven individuals carrying the $\mathrm{CW}^{*} 0701-\mathrm{B} 8$ haplotype also carried CD6. HLA$\mathrm{Cw}^{*} 0702$, the other frequently observed $\mathrm{Cw} 7$ allele, was linked with CD5 without exception, either on the B7- $\mathrm{Cw}^{*} 0702$ or the B39$\mathrm{Cw}^{*}$ 0702-haplotype. Other HLA-haplotypes yielding strong linkage disequilibrium with CD5 were Cw*04-B35 (100\%) and Cw*0303-B62 (80\%); and $\mathrm{CW}^{*} 08$-B65 was found linked without exception with CD1a.

\section{Association of HLA and CD alleles with psoriasis}

Exclusive HLA disease association studies were not the aim of this investigation. As the families selected for this study were chosen on the basis of the presence of psoriasis-associated HLA haplotypes, the overrepresentation of distinct HLA alleles was to be expected. The TDT was applied to clarify whether CD yields stronger association with psoriasis than HLA.

As expected, the TDT confirmed the strong association of psoriasis with HLA alleles belonging to the psoriasis-related HLA haplotypes. $\mathrm{CD} 2$, being linked with both $\mathrm{Cw}^{*} 06-\mathrm{B} 37$ and $\mathrm{Cw}^{*} 06$ B57, presented a relative risk higher than that observed for HLACw*06 (3.4 vs. 2.5, not significant). In contrast, neither CD3, demonstrating 100\% linkage disequilibrium with $\mathrm{Cw}^{*} 06$-B13, nor CD1, associated with the $\mathrm{Cw} * 08$-B65-haplotype, demonstrated any association with disease (Table 9).

Of the amino acid variants encoded by the various $\mathrm{CD}$ gene alleles, none reached the transmission ratio or $P$-value observed for the CD2 allele (Table 10). In addition, no evidence of an association between psoriasis and CD1, the only CD allele which carries all of the putative disease associated amino acid residues, was identified.

\section{Discussion}

Recent genetic linkage studies identified the major susceptibility gene for familial psoriasis, PSORS1, as being located within the human $\mathrm{MHC}$ in close proximity to the HLA-B/C region (5-8). PSORS1 is found in strong linkage disequilibrium with distinct HLA alleles which form the specific, psoriasis-associated haplotypes $(7,9)$. These haplotypes must not necessarily be positive for Cw6 (17, 22), although Cw6 remains the strongest marker for psoriasis identified so far.

Due to its function and location, $\mathrm{CD}$ appears as a strong candidate for psoriasis susceptibility. In order to investigate the polymorphisms of $\mathrm{CD}$ and to develop new insights into its relationship with familial psoriasis, the $\mathrm{CD}$ sequences were more extensively investigated. The aims of this study were to: i) identify additional

\begin{tabular}{|c|c|c|c|c|c|}
\hline & \multicolumn{5}{|l|}{ HLA-locus } \\
\hline & A & $\mathrm{Cw}$ & B & DRB1 & DQB1 \\
\hline$\chi^{2}$ & 114.77 & 195.02 & 248.11 & 85.03 & 55.63 \\
\hline df & 60 & 84 & 120 & 78 & 36 \\
\hline$P$-value & 0.000027 & $<0.0000000001$ & $<0.0000000001$ & 0.27 & 0.02 \\
\hline
\end{tabular}


Observed CD / HLA-haplotypes and the respective $\Delta_{\mathrm{r}}$ values (in brackets). Only haplotypes observed at least three times and only $\Delta_{\mathrm{r}}>0.1$ are reported

\begin{tabular}{|c|c|c|c|c|c|c|c|c|c|}
\hline & Allele & $n$ & $\begin{array}{l}\text { CD1a } \\
(n=17)\end{array}$ & $\begin{array}{l}\text { CD1b } \\
(n=1)\end{array}$ & $\begin{array}{l}\text { CD2 } \\
(n=11)\end{array}$ & $\begin{array}{l}\text { CD3 } \\
(n=3)\end{array}$ & $\begin{array}{l}\text { CD4 } \\
(n=6)\end{array}$ & $\begin{array}{l}\text { CD5 } \\
(n=16)\end{array}$ & $\begin{array}{l}\text { CD6 } \\
(n=12)\end{array}$ \\
\hline \multirow[t]{4}{*}{ HLA-A } & $\mathrm{A} 1$ & 17 & - & - & $5(0.27)$ & - & - & - & $9(0.66)^{\mathrm{c}}$ \\
\hline & $\mathrm{A} 2$ & 21 & $7(0.14)$ & - & $5(0.20)$ & - & - & - & - \\
\hline & A24 & 5 & $3(0.46)$ & - & - & - & - & - & - \\
\hline & A3 & 9 & - & - & - & - & - & $8(0.85)^{c}$ & - \\
\hline \multirow[t]{8}{*}{ HLA-Cw } & $* 02$ & 3 & $3(1.00)$ & - & - & - & - & - & - \\
\hline & *0303 & 7 & $3(0.23)$ & - & - & - & - & $4(0.43)$ & - \\
\hline & *0304 & 9 & $5(0.40)$ & - & - & - & - & - & - \\
\hline & $* 04$ & 3 & - & - & - & - & - & $3(1.00)$ & - \\
\hline & $* 06$ & 13 & - & - & $9(0.77)^{d}$ & $3(1.00)^{a}$ & - & - & - \\
\hline & *0701 & 10 & - & - & - & - & - & - & $8(0.76)^{d}$ \\
\hline & *0702 & 9 & - & - & - & - & - & $9(1.00)^{d}$ & - \\
\hline & $* 08$ & 3 & $3(1.00)$ & - & - & - & - & - & - \\
\hline \multirow[t]{9}{*}{ HLA-B } & B7 & 9 & - & - & - & - & - & $8(0.85)^{c}$ & - \\
\hline & B8 & 7 & - & - & - & - & - & - & $7(1.00)^{d}$ \\
\hline & B13 & 4 & - & - & - & $3(1.00)^{c}$ & - & - & - \\
\hline & B35 & 4 & - & - & - & - & - & $3(0.67)$ & - \\
\hline & B37 & 3 & - & - & $3(1.00)^{a}$ & - & - & - & - \\
\hline & B57 & 4 & - & - & $4(1.00)^{c}$ & - & - & - & - \\
\hline & $\mathrm{B} 60$ & 6 & $3(0.33)$ & - & - & - & - & - & - \\
\hline & B62 & 7 & $3(0.23)$ & - & - & - & - & $4(0.43)$ & - \\
\hline & B65 & 3 & $3(1.00)$ & - & - & - & - & - & \\
\hline HLA- & $* 01$ & 5 & - & - & - & - & - & $3(0.47)$ & - \\
\hline \multirow[t]{6}{*}{ DRB1 } & *0301 & 5 & - & - & - & - & - & - & $3(0.51)$ \\
\hline & $* 08$ & 6 & - & - & - & - & $3(0.45)^{a}$ & - & - \\
\hline & $* 11$ & 7 & - & - & $3(0.31)$ & - & - & - & - \\
\hline & $* 13$ & 10 & $4(0.19)$ & - & - & - & - & - & $3(0.14)$ \\
\hline & $* 07$ & 9 & - & - & $3(0.20)$ & - & - & - & - \\
\hline & $* 15$ & 4 & - & - & - & - & - & $4(1.00)^{c}$ & - \\
\hline HLA- & $* 02$ & 10 & - & - & - & - & - & - & $4(0.27)$ \\
\hline \multirow[t]{5}{*}{ DQB1 } & *0301 & 15 & - & - & $4(0.18)$ & - & - & - & - \\
\hline & *0302 & 10 & $4(0.19)$ & - & - & - & - & - & - \\
\hline & *0303 & 4 & - & - & $3(0.70)$ & - & - & - & - \\
\hline & $* 04$ & 5 & - & - & - & - & $3(0.56)^{a}$ & - & - \\
\hline & $* 05$ & 6 & - & - & - & - & - & $3(0.34)$ & - \\
\hline
\end{tabular}

$n$ : Total number of HLA and CD alleles observed, respectively. a: $P<0.01,{ }^{b}: P<0.001,{ }^{c}: P<0.0001,{ }^{d}: P<0.00001$

Table 7

polymorphic sites within the $\mathrm{CD}$ sequence; ii) clarify whether these polymorphic sites are sporadic or belong to conserved alleles; iii) analyze linkage disequilibrium between these conserved alleles and the alleles of the HLA system; and, finally, iv) investigate whether $\mathrm{CD}$ alleles might play a role in the genetics of psoriasis.
For that purpose, the second exon of the $\mathrm{CD}$ was completely sequenced from eighty six fully HLA-typed individuals descending form 13 families with high incidence of familial psoriasis. This approach offered several advantages: i) it provided information regarding all bases within the amplified region, and any heterozygous 


\begin{tabular}{|c|c|c|c|c|c|c|c|c|}
\hline \multicolumn{2}{|l|}{ HLA } & \multicolumn{7}{|c|}{$C D$ gene } \\
\hline $\mathrm{Cw}$ & B & CD1a & CD1b & $\mathrm{CD} 2$ & $\mathrm{CD} 3$ & CD4 & CD5 & $\mathrm{CD} 6$ \\
\hline \multirow[t]{3}{*}{ *02 } & B7 & 1 & - & - & - & - & - & - \\
\hline & B27 & 1 & - & - & - & - & - & - \\
\hline & B61 & 1 & - & - & - & - & - & - \\
\hline \multirow[t]{2}{*}{ *0303 } & B55 & 2 & - & - & - & - & - & - \\
\hline & B62 & 1 & - & - & - & - & 4 & - \\
\hline \multirow[t]{3}{*}{ *0304 } & B58 & - & - & - & - & - & - & 1 \\
\hline & $\mathrm{B} 60$ & 3 & 1 & 1 & - & - & - & 1 \\
\hline & B62 & 2 & - & - & - & - & - & - \\
\hline$* 04$ & B35 & - & - & - & - & - & 3 & - \\
\hline *05 & B44 & - & - & - & - & - & - & 1 \\
\hline \multirow[t]{5}{*}{ *06 } & B13 & 1 & - & - & 3 & - & - & - \\
\hline & B37 & - & - & 3 & - & - & - & - \\
\hline & B38 & - & - & 1 & - & - & - & - \\
\hline & B50 & - & - & 1 & - & - & - & - \\
\hline & B57 & - & - & 4 & - & - & - & - \\
\hline \multirow[t]{3}{*}{ *0701 } & B18 & - & - & - & - & 1 & - & 1 \\
\hline & B49 & - & - & 1 & - & - & - & - \\
\hline & B8 & - & - & - & - & - & - & 7 \\
\hline \multirow[t]{2}{*}{ *0702 } & B39 & - & - & - & - & - & 1 & - \\
\hline & B7 & - & - & - & - & - & 8 & - \\
\hline *0704 & B44 & 1 & - & - & - & - & - & - \\
\hline *08 & B65 & 3 & - & - & - & - & - & - \\
\hline \multirow[t]{3}{*}{ *1203 } & B18 & - & - & - & - & 1 & - & - \\
\hline & B35 & - & - & - & - & 1 & - & - \\
\hline & B38 & - & - & - & - & - & - & 1 \\
\hline *1301 & B51 & - & - & - & - & 1 & - & - \\
\hline *15 & B52 & - & - & - & - & 1 & - & - \\
\hline *1601 & B44 & 1 & - & - & - & - & - & - \\
\hline *1602 & B51 & - & - & - & - & 1 & - & - \\
\hline
\end{tabular}

TDT results between CD gene alleles and psoriasis. Results are compared to those obtained for HLA alleles found on the same ancestral haplotypes

\begin{tabular}{|c|c|c|c|c|c|c|c|c|c|c|c|}
\hline \multicolumn{2}{|c|}{ Haplotype } & \multicolumn{2}{|l|}{$C D$} & \multicolumn{2}{|c|}{ HLA-CW } & \multicolumn{2}{|l|}{ HLA-B } & \multicolumn{2}{|l|}{ DRB1 } & \multicolumn{2}{|l|}{ DQB1 } \\
\hline$C D$ & HLA & $\mathrm{t} / \mathrm{nt}$ & prob. & $\mathrm{t} / \mathrm{nt}$ & prob. & $\mathrm{t} / \mathrm{nt}$ & prob. & $\mathrm{t} / \mathrm{nt}$ & prob. & $\mathrm{t} / \mathrm{nt}$ & prob. \\
\hline CD1 & Cw*08-B65-DRB1*01-DQB1*05 & $10 / 9$ & 0.5 & $6 / 2$ & 0.14 & $6 / 2$ & 0.14 & $5 / 3$ & 0.26 & $7 / 1$ & 0.035 \\
\hline $\mathrm{CD} 2$ & Cw*06-B57-DRB1*07-DQB1*0303 & $17 / 5$ & 0.0085 & $20 / 8$ & 0.018 & $11 / 2$ & 0.011 & $11 / 5$ & 0.11 & $7 / 1$ & 0.035 \\
\hline $\mathrm{CD} 2$ & Cw*06-B37-DRB1*07-DQB1*02 & $17 / 5$ & 0.0085 & $20 / 8$ & 0.018 & $3 / 1$ & 0.31 & $11 / 5$ & 0.11 & $5 / 12$ & 0.93 \\
\hline CD3 & Cw*06-B13-DRB1*07-DQB1*02 & $2 / 2$ & 0.5 & $20 / 8$ & 0.018 & $3 / 4$ & 0.50 & $11 / 5$ & 0.11 & $5 / 12$ & 0.93 \\
\hline CD4 & none & $2 / 5$ & 0.77 & - & - & - & - & - & - & - & - \\
\hline CD5 & Cw*0702-B7-DRB1*15-DQB1*06 & $5 / 13$ & 0.95 & $4 / 9$ & 0.87 & $3 / 11$ & 0.97 & $3 / 4$ & 0.5 & $7 / 7$ & 0.5 \\
\hline CD6 & $\mathrm{Cw} * 0701-\mathrm{B} 8-\mathrm{DRB} 1 * 03-\mathrm{DQB} 1 * 02$ & $10 / 12$ & 0.58 & $9 / 11$ & 0.59 & $7 / 10$ & 0.69 & $2 / 9$ & 0.97 & $5 / 12$ & 0.93 \\
\hline
\end{tabular}

Table 9 
TDT results between psoriasis and amino acid residues of the CD gene

\begin{tabular}{|c|c|c|c|}
\hline Residue & CD alleles & $\mathrm{t} / \mathrm{nt}$ & prob. \\
\hline $\operatorname{Ser}_{143}$ & $\mathrm{CD} 1, \mathrm{CD} 2, \mathrm{CD} 3, \mathrm{CD} 4, \mathrm{CD} 6$ & $13 / 5$ & 0.048 \\
\hline $\operatorname{ser}_{153}$ & $\mathrm{CD} 1, \mathrm{CD} 2, \mathrm{CD} 3, \mathrm{CD} 4, \mathrm{CD} 5$ & $12 / 10$ & 0.42 \\
\hline ser $_{202}$ & CD1 & $9 / 8$ & 0.5 \\
\hline $\operatorname{ser}_{401}$ & $\mathrm{CD} 1, \mathrm{CD} 2$ & $2 / 2$ & 0.5 \\
\hline $\operatorname{ser}_{408}$ & $\mathrm{CD} 1, \mathrm{CD} 2, \mathrm{CD} 4, \mathrm{CD} 5, \mathrm{CD} 6$ & $5 / 2$ & 0.23 \\
\hline $\operatorname{ser}_{410}$ & CD1, CD2, CD3, CD4 & $22 / 12$ & 0.061 \\
\hline $\operatorname{asp}_{527}$ & $\mathrm{CD} 1, \mathrm{CD} 2, \mathrm{CD} 3, \mathrm{CD} 4, \mathrm{CD} 6$ & $13 / 5$ & 0.048 \\
\hline
\end{tabular}

Table 10

sites could be confirmed by sequence comparisons with first grade relatives; ii) the HLA haplotype information allowed the designation of the phase of inheritance of each MHC haplotype and made it easier to assign the nucleotides of the polymorphic sites to alleles; and iii) finally, for association analysis, a control based upon inheritance was possible, avoiding problems due to mixed sample population (23).

Using this approach, we identified seven different CD gene alleles encoding six distinct proteins. We have also placed five of these alleles on well-known ancestral haplotypes defined by HLA-C and -B. Finally, we provide strong evidence for an allelic association between the CD2 allele and PSORS1.

Of the nine polymorphic sites already published $(13,15)$, we were able to confirm all but one. This dimorphic site, located at nt. 1240 (val/gly ${ }_{409}$ ), was found in a Japanese cohort on the Cw3-B35 haplotype (15). This haplotype occurs only rarely within the Caucasian population (24), and was not observed in our study group.

None of the alleles defined by us encoded completely either for the CD sequence (13), or for the S-gene product (10). The deletion of a guanosine at nt. 1515 in the S-gene sequence was not observed either in our study nor by others (13), and is therefore probably due to a sequencing error. In contrast, we were able to confirm all residues described for the $\mathrm{CD}$ sequence on polymorphic sites; however, not on one distinct allele (Table 5). We therefore assume that the described $\mathrm{CD}$ sequence might be a $\mathrm{CD}$ allele not present in our study.

The alleles of the $\mathrm{CD}$ gene yielded strong linkage disequilibrium with HLA. Due to the close physical proximity of the $\mathrm{CD}$ to the $\mathrm{HLA}-\mathrm{B} / \mathrm{C}$ region, it would be quite reasonable to expect that the strongest linkage disequilibrium would be observed at these two loci. However, since the CD is located telomeric of HLA-C, a stronger linkage disequilibrium with HLA-C than with HLA-B would be an- ticipated, especially since HLA-C is the less polymorphic locus. In contrast, some of the HLA-C alleles were found linked with more than one CD allele, whereas some of the HLA-B alleles and the respective HLA-B/C haplotypes yielded much stronger linkage disequilibrium with the $S$-gene. For example, $\mathrm{Cw}^{*} 06$ was found in cis with CD1a, CD2 and CD3, whereas Cw*06-B57 and Cw*06-B37 were found in linkage with CD2 without exception. Thus, linkage disequilibrium of CD with HLA seems to be of a haplotype- rather than of a locus-specific form.

Most of the common HLA-B/C haplotypes present in our study showed linkage disequilibrium with only one distinct $\mathrm{CD}$ allele. Such haplotypes were CD5-Cw*0701-B8, CD6-Cw*0702-B7, CD5$\mathrm{CW}^{*} 04-\mathrm{B} 35$ and $\mathrm{CD} 2-\mathrm{Cw}^{*} 06-\mathrm{B} 37 / \mathrm{B} 57$. Although the overall linkage disequilibrium of the CD-gene with HLA-class II loci was not significant, some of the HLA-class II alleles belonging to extended haplotypes (i.e. DQB1*0303, DRB1*15, DRB1*0301) still yielded remarkably linkage disequilibrium with the respective $\mathrm{CD}$ allele. These data provide strong evidence that the $\mathrm{CD}$ polymorphism is not sporadic but rather old and well conserved.

In a preliminary analysis of the possible role of the CD-alleles in the genetics of psoriasis, we investigated whether any $\mathrm{CD}$ allele or polymorphic site within the $\mathrm{CD}$ sequence yielded stronger diseaseassociation than the HLA alleles. Indeed, the most significant association was observed for CD, i.e. the CD2 allele. Due to the extensive linkage disequilibrium of $\mathrm{CD} 2$ with both $\mathrm{Cw6}-\mathrm{B} 57$ and $\mathrm{Cw6}$ B37, a relative risk higher than that of HLA-Cw6 was observed (3.4 vs. 2.5), although without being significant. In addition, CD2 was also found linked with two more rare $\mathrm{Cw6}$-positive haplotypes (Cw6B38 and Cw6-B50) which segregate with disease. B57 which demonstrates the highest relative risk observed in the study (5.5), occurred less frequently in psoriatics and is therefore of less significance than CD2.

Whereas these results indicate a strong potential role for CD in the pathogenesis of psoriasis, other observations conflict with this postulate. Certain families included in the study presented the Cw6negative haplotype Cw8-B65 which segregates with disease. Assuming direct involvement of $\mathrm{CD}$, linkage of this haplotype (and of other HLA-haplotypes found in Cw6-negative psoriatics) with CD2 would be expected. However, the $\mathrm{CW}^{*} 08$-B65 haplotype was found linked with the CD1 allele, which demonstrated no association, as compared to $\mathrm{B} 65$ and $\mathrm{Cw} * 08$ which both yielded a relative risk of three (6/2). CD1 differs from CD2 only in one amino acid at position 202. Ser $_{202}$ is however, specific for CD1, whereas phe 202 was found not only in $\mathrm{CD} 2$ but in all other $\mathrm{CD}$ alleles; indicating that position 202 is not relevant for psoriasis. Concordantly, none of the other polymorphic residues demonstrated any strong association. These results are in accordance with those reported by Ishihara et al. (15), 
where the association of psoriasis with distinct residues of the $\mathrm{CD}$ was also not observed. This indicates that the association of the $\mathrm{CD}$ alleles with psoriasis might be only due to linkage disequilibrium.

In summary, our results indicate that the $\mathrm{CD} 2$ allele of the $\mathrm{CD}$ seems to be at least an excellent marker for psoriasis, probably better than HLA. Furthermore, CD is thus far the only skin-specific protein encoded within the psoriasis susceptibility region in the MHC and its functionality makes it a strong candidate for disease susceptibility. Further investigations of the functional and regulatory differences of the $\mathrm{CD}$ alleles will need to demonstrate whether this molecule is indeed involved in the pathogenetics of psoriasis.

\section{Note added in proof:}

After submission of this manuscript, a research letter on the same topic was published (Allen MH, Veal C, Faassen A, Powis SH, Vaughan RW, Trembath RC, Barker JNWN. Lancet 1999: 353: 1589). In that study, an allele defined by intragenic bi-allelic polymorphisms at nt. 619 (t) , nt. 1240 (g) , and nt. 1243 (c), was identified to be highly significant associated with psoriasis. Although Allen et al. did not investigate all the polymorphic sites described in our paper, the respective nucleotides are identical with those of $\mathrm{CD} 2$, indicating that both psoriasis-associated alleles are indeed most probably identical.

\section{References}

1. Christophers E, Sterry W. Psoriasis. In: Fitzpatrick TB, Eisen AZ, Wolff K, Freedberg IM, Austen KF, eds. Dermatology in General Medicine. New York: McGrawHill, 1993: 489-514.

2. Henseler T, Christophers E. Psoriasis of early and late onset: characterization of two types of psoriasis vulgaris. J Am Acad Dermatol 1985: 13: 450-6.

3. Christophers E. The immunopathology of psoriasis. Int Arch Allergy Immunol 1996: 110: 199-206.

4. Elder JT, Nair RP, Guo SW, Henseler T, Christophers E, Voorhees JJ. The genetics of psoriasis. Arch Dermatol 1994: 130: 216-24.

5. Trembath RC, Clough RL, Rosbotham JL et al. Identification of a major susceptibility locus on chromosome $6 \mathrm{p}$ and evidence for further disease loci revealed by a two stage genome-wide search in psoriasis. Hum $\mathrm{Mol}$ Genet 1997: 6: 813-20.

6. Nair RP, Henseler T, Jenisch S et al. Evidence for two psoriasis susceptibility loci (HLA and 17q) and two novel candidate regions (16q and 20p) by genomewide scan. Hum Mol Genet 1997: 6: 134956.

7. Jenisch S, Henseler T, Nair RP et al. Linkage analysis of human leukocyte antigen (HLA) markers in familial psoriasis: strong disequilibrium effects provide evidence for a major determinant in the HLA-B/-C region. Am J Hum Genet 1998: 63: 191-9.
8. Leder RO, Mansbridge JN, Hallmayer J, Hodge SE. Familial psoriasis and HLA-B: unambiguous support for linkage in 97 published families. Hum Hered 1998: 48: 198-211.

9. Jenisch S, Westphal E, Nair RP et al. Linkage disequilibrium analysis of familial psoriasis: identification of multiple disease-associated $\mathrm{MHC}$ haplotypes. Tissue Antigens 1999: 53: 135-46.

10. Zhou Y, Chaplin DD. Identification in the HLA class I region of a gene expressed late in keratinocyte differentiation. Proc Natl Acad Sci U S A 1993: 90: 9470-4.

11. Chaplin DD, Zhou Y. HLA-linked skin disease: classical HLA genes or novel genes within HLA? J Invest Dermatol 1995: 104: $37 \mathrm{~S}$.

12. Haftek M, Simon M, Kanitakis J et al. Expression of corneodesmosin in the granular layer and stratum corneum of normal and diseased epidermis. $\mathrm{Br} \mathrm{J}$ Dermatol 1997: 137: 864-73.

13. Guerrin M, Simon M, Montezin M, Haftek M, Vincent C, Serre G. Expression cloning of human corneodesmosin proves its identity with the product of the $\mathrm{S}$ gene and allows improved characterization of its processing during keratinocyte differentiation. J Biol Chem 1998: 273 : 22640-7.
14. Simon M, Montezin M, Guerrin M, Durieux JJ, Serre G. Characterization and purification of human corneodesmosin, an epidermal basic glycoprotein associated with corneocyte-specific modified desmosomes. J Biol Chem 1997: 272: 31770 6.

15. Ishihara M, Yamagata N, Ohno S et al. Genetic polymorphisms in the keratin-like $\mathrm{S}$ gene within the human major histocompatibility complex and association analysis on the susceptibility to psoriasis vulgaris. Tissue Antigens 1996: 48: $182-6$.

16. Nair RP, Guo SW, Jenisch S et al. Scanning Chromosome 17 for Psoriasis Susceptibility: Lack of Evidence for a Distal 17q Locus. Hum Hered 1995: 45: 219 30.

17. Jenisch S, Nair RP, Henseler T et al. Association of type I psoriasis with the Cw*0802-DRB1*0102-DQB1*0501 haplotype in north-American multiplex families. In: Charron D, ed.. Genetic Diversity of HLA: Functional and Medical Implication. Paris: EDK, 1997: 712-5.

18. Darke C, Dyer P. Clinical HLA typing by cytotoxicity. In: Dyer P, Middleton D, eds. Histocompatibility testing: A Practical Approach. Oxford: Oxford University Press, 1993: 51-80. 
19. Saiki RK, Walsh PS, Levenson $\mathrm{CH}$, Erlich HA. Genetic analysis of amplified DNA with immobilized sequence-specific oligonucleotide probes. Proc Natl Acad Sci U S A 1989: 86: 6230-4.

20. Baur MP, Danilovs JA. Population Analysis of HLA-A,B,C,DR, and Other Genetic Markers. In: Terasaki PI, ed.

Histocompatibility Testing 1980. Los Angeles, CA: UCLA Tissue Typing Laboratory, 1980: 955-93.
21. Spielman RS, McGinnis RE, Ewens WJ. Transmission test for linkage disequilibrium: the insulin gene region and insulin-dependent diabetes mellitus (IDDM). Am J Hum Genet 1993: 52: 506-16.

22. Nakagawa H, Akazaki S, Asahina A et al. Study of HLA class I, class II and complement genes (C2, C4A, C4B and $\mathrm{BF}$ ) in Japanese psoriatics and analysis of a newly-found high-risk haplotype by pulsed field gel electrophoresis. Arch Dermatol Res 1991: 283: 281-4.
23. Thomson G. HLA disease associations: models for the study of complex human genetic disorders. Crit Rev Clin Lab Sci 1995: 32: 183-219.

24. Imanishi T, Akaza T, Kimura A, Tokunaga K, Gojobori T . Allele and haplotype frequencies for HLA and complement loci in various ethnic groups. In: Tsuji $\mathrm{K}$, Aizawa M, Sasazuki K, eds. HLA 1991. Vol. 1. New York: Oxford Science Publications, 1992: 1065-220. 